\title{
Use of a device for bone allograft channeling in an experiment with rabbits: Narrative review
}

\author{
Alexandr Rudenko ${ }^{1,2}$, Berik Tuleubaev ${ }^{1,2}$, Nurettin Heybeli ${ }^{1,2}$ \\ ${ }^{I}$ Department of Surgical Diseases, Karaganda Medical University, Karaganda, Kazakhstan \\ ${ }^{2}$ Department of Emergency Trauma, Multidisciplinary Regional Center of Traumatology and Orthopedics named after Professor Kh.Zh.Makazhanov,
} Karaganda, Kazakhstan

Received: 2021-09-27.

Accepted: 2022-02-07

This work is licensed under a Creative Commons Attribution 4.0 International License

\section{J Clin Med Kaz 2022; 19(1):65-69}

Corresponding author: Alexandr Rudenko.

E-mail: uda4a-@mail.ru; ORCID: 0000-0002-5354-8040

\section{Abstract}

Aim: to investigate the method of antibiotic impregnation of the femoral bone head (prepared according to the Marburg bone bank system) using a patented device designed for uniform channeling followed by deep soaking of the bone graft spongy tissue with drug solutions.

Materials and methods: Femoral bone heads prepared according to the Marburg bone bank system and impregnated with gentamicin were used in the experiment. Four groups were formed depending on the impregnation method. A whole femoral head was used in groups I and II, a perforated femoral head - in groups III and IV. In groups I and III, antibiotic impregnation was carried out simultaneously with heat treatment of the femoral heads, in groups II and IV - after heat treatment. The perforated, treated, gentamicin-soaked femoral bone head was compared to the "PerOssal" beads soaked in gentamicin. The degree of influence on the S. aureus strains was determined using the agar diffusion method.

Results and conclusion: The results indicate the presence of antimicrobial activity in the antibiotic-impregnated allograft in all groups. However, the growth inhibition zone was lower in groups I and III. The antimicrobial activity of gentamicin in the I and III was lower than in the groups II and IV. An increase in the diffusion of the antibiotic is facilitated by the perforation of the bone allograft according to the developed method using a patented device. It has been established that perforation and soaking of the bone allograft in an antibiotic solution increases the antibiotic impregnation of the bone graft. osteomyelitis

Key words: antibiotic prophylaxis, arthroplasty, implant,

\section{Introduction}

Currently, infectious complications after chronic osteomyelitis are an important problem in traumatology and orthopedics, which should be paid attention to [1]. The use of bone allograft avoids fractures and chronic pain syndromes at the area of donor material collection. The literature often describes the impregnation of a bone graft by manual stirring, shaking, placing the bone graft into antibiotic-containing solutions for a certain period, as well as physical methods (iontophoresis) [2-4]. A number of researchers describe the use of bone allografts as carriers for antibiotics. Thus, E. Wits et al. found that ground bone grafts impregnated with antibiotics are effective for a variety of clinical situations - they store and release large amounts of aminoglycosides and vancomycin [5].
It is known that, grafts in the form of allo-, auto-, or heterotransplants are used in osteoplastic operations [6, 7]. When filling defects in bone tissue, a backbone from an organic matrix is required, on which new bone tissue is formed. The use of bone chips or whole massive bone grafts to fill large bone defects showed that partial resorption occurs, and the bone defect does not completely heal [8]. Therefore, the allograft must be created in the form of a maximally porous spongy skeleton, along which the restoration of bone tissue will be more favorable, and the penetration of liquid with antiseptic substances (antibiotic solutions) will provide better graft impregnation [9].

Orthopedic awl, described in the manual of G. Ye. Ostroverkhov, is a known device [10]. The device consists of an oblong handle and a stylet with a tapered point. This device can form channels in the graft in order to improve 
the penetration of drugs into the graft depth. However, the use of this device has the following disadvantages:

1. Irrationality of the oblong shape of the orthopedic awl handle, continuing along the vector of its stylet. The tight grip of the instrument during the formation of the bone canal causes bending and overstraining of the muscles of the surgeon's hand with dulling of his "muscular feeling". This leads to an uneven distribution of canals over the allograft mass, which implies impregnation of the spongy bone allograft with liquid solutions of different saturation [11]. As a result, zones are formed with the absence of the drug, or with the drug content less than the required level. Also, in the place of possible intersection of the canals, a cavity is formed, which will create a defect in the neoplasm of bone tissue.

2. The absence of indicative marks on the awl stylet causes insufficient visual control over the depth of the canals.

3. Long time of channel formation.

4. A "cleaver effect" is possible, in which wedging and cracking of the bone occurs.

For this study, a device was created with which it is possible to quickly and conveniently form uniformly distributed canals in the bone allograft array. The channels are designed for uniform impregnation of bone allograft spongiosa with solutions, as well as for increasing the allograft surface area for bone tissue neoplasm.

Figure 1 - Utility model patent "Device for bone allograft perforation"

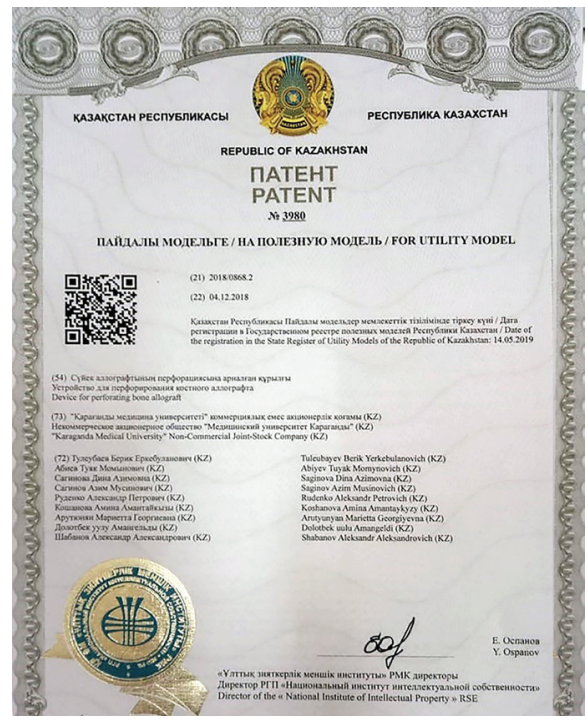

The utility model relates to medical technology, namely to traumatology and orthopedics, and is intended for uniform channeling with subsequent deep soaking of the spongy tissue of the bone graft with solutions of medicinal substances. The device is patented, utility model patent No. 3980 [12] (Figure 1).

\section{Materials and methods}

The new device was used for bone allograft channeling, followed by the impregnation of medicinal substances into the bone tissue. The device consists of a rectangular structure made of metal, $50 \mathrm{~mm}$ wide, $50 \mathrm{~mm}$ high and $100 \mathrm{~mm}$ long. Threaded holes for brackets are available on two side walls, which is necessary for stable fixation of the bone allograft inside the base. The other two walls have through channels located at the same distance from each other over the entire surface of the wall. Channels $10 \mathrm{~mm}$ thick are required to prevent drill deflection. Multiple through channels at the same distance from each other are formed on two perpendicular sides in the bone allograft, they are intended for subsequent uniform bone tissue impregnation (Figures 2-4).

Figure 2 - General view of a device for bone allograft channeling with subsequent impregnation of drugs into bone tissue

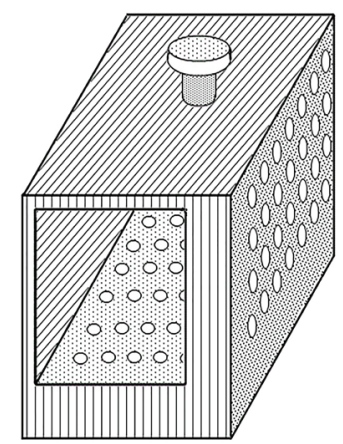

Figure 3 - Side view of a device for bone allograft channeling with subsequent impregnation of drugs into the bone tissue

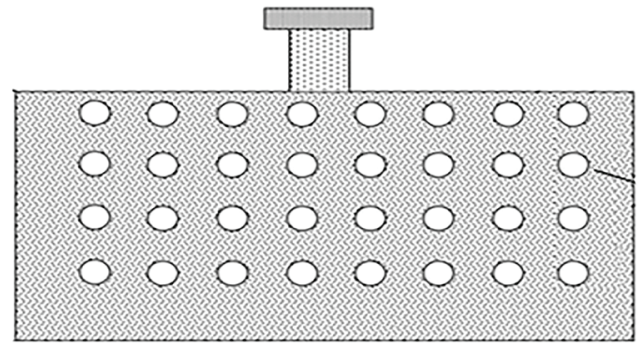

Figure 4 - Sectional view of a device for bone allograft channeling with subsequent impregnation of drugs into the bone tissue with a fixed graft

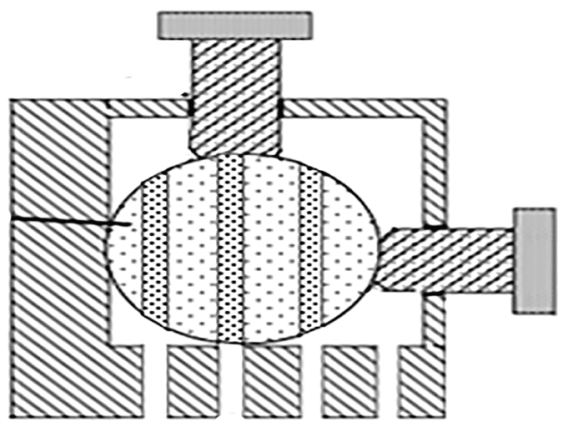

Removed femoral bone head is used after hip arthroplasty for the manufacture of an allograft matrix. The formation of channels using a drill is carried out in the head after appropriate mechanical treatment and boiling in order to deeply impregnate with drugs and create a skeleton. The optimal conditions for the formation of bone tissue and scaffold are combined if the distance between adjacent canals is the same. After the formation of canals in the allograft, further processing is carried out in the Lobator device according to the Marburg system (EP0584484A1. 02.03.1994. Verfahren und Einrichtung zum Desinfizieren von Knochentransplantaten, insbesondere von humanen SpongiosaTransplantaten Harald Priv. Dozent Dr. KnaeplerGarrel Thomas Dr. Von.). Then the allograft is placed in a sterile bag with an antiseptic liquid and sealed.

The method of device using is as follows. The bone graft is fixed with two bolt brackets in the device. Channels are formed by the drill according to a template (channels for a drill, located 
at the same distance from each other over the entire surface of the two perpendicular walls of the device). Then the graft is washed in saline to clean the canals from bone chips. Next, the antibiotic is impregnated by boiling and soaking with the antibiotic in a Lobator device according to the Marburg system.

Figure 5 - Top view of a device for bone allograft channeling with subsequent impregnation of drugs into the bone tissue with femoral bone head fixed in it

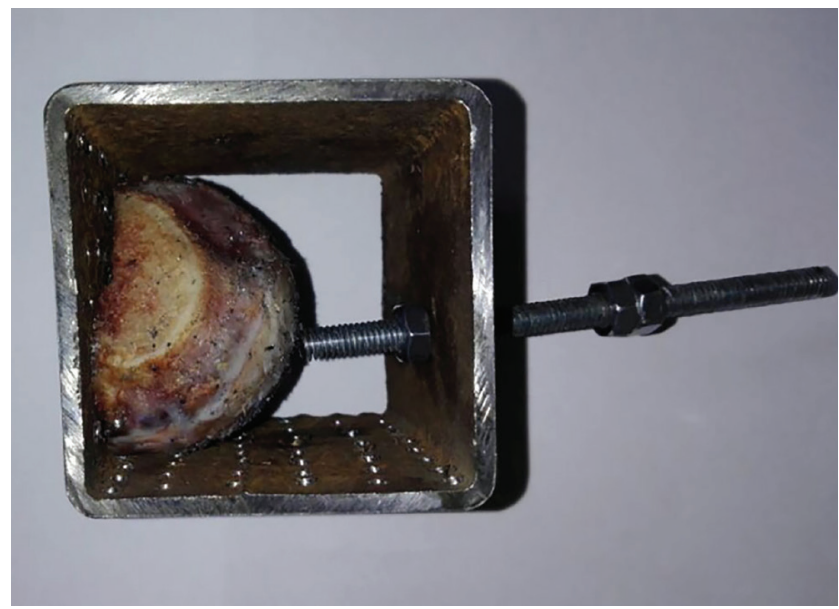

Figure 7 - Side view of a device for bone allograft channeling. The holes with a diameter of $3.2 \mathrm{~mm}$ for perforation are visible on the side wall

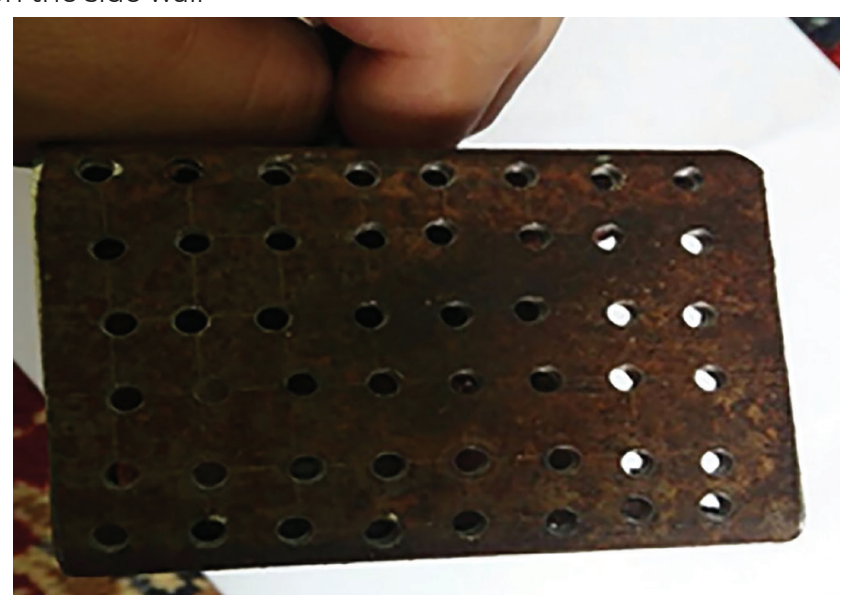

The criteria for excluding heads from the experiment: the presence of severe sclerosis and damage. There were various damages of the femoral bone head. Basically, these were focal lesions of the cartilaginous tissue typical for cocarthrosis of the III-IV degree. The average diameter of the femoral bone heads was $53 \mathrm{~mm}$. The treated head was placed in a special cartridge with $330 \mathrm{ml}$ of saline solution. The entire surface of the graft was placed in saline solution. The head cartridge was processed for 94 minutes on a Lobator apparatus (Figure 8).

The temperature in the center of the allograft was 82.5 ${ }^{\circ} \mathrm{C}$. After processing the grafts on the Lobator apparatus, the femoral bone heads were sterile and completely ready for further work. Gentomycin was chosen as an antibiotic for impregnation because it showed good thermal stability properties.

Bone grafts were divided into 4 groups depending on the impregnation method:

Group I: whole head, treated on the Lobator sd-2 apparatus with the addition of gentamicin;
The bone graft was taking in patients after hip arthroplasty using a special instrument, namely electrocutter. The femoral bone heads were cleaned of cartilaginous tissue. Femoral bone head perforation was performed using an original bone allograft perforation device (Figures 5-7).

Figure 6 - Side view of a device for bone allograft channeling with subsequent impregnation of drugs into the bone tissue with femoral bone head fixed in it

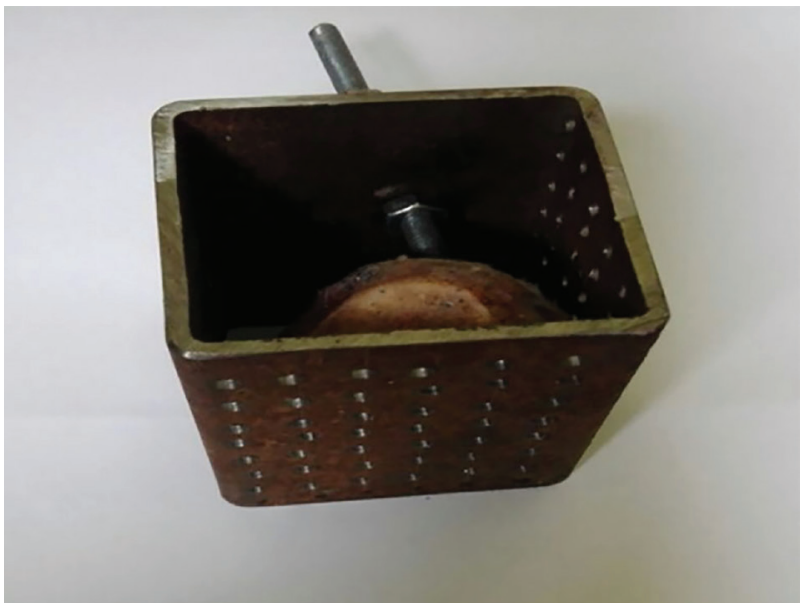

Figure 8 - Antibiotic treatment of the whole femoral bone head in the Lobator sd-2 system
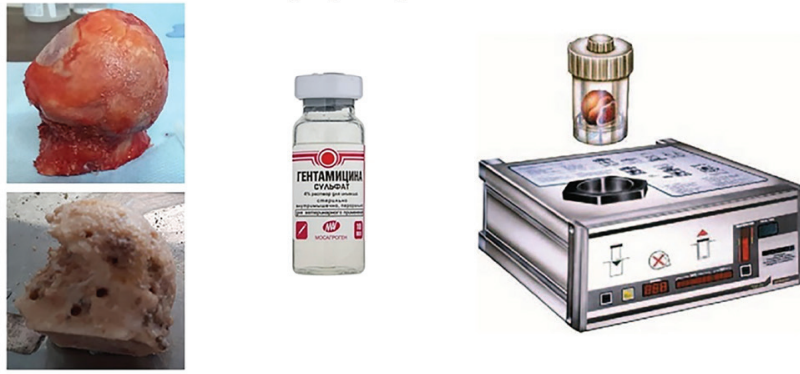

Group II: after treatment on the Lobator sd-2 apparatus, the graft was placed in a gentamicin solution with an exposure time of 60 minutes;

Group III: the graft was perforated according to the developed technique, then treatment was carried out on the Lobator sd-2 apparatus with the addition of gentamicin;

Group VI: the graft was perforated according to the developed technique, then treatment was carried out on the Lobator sd-2 apparatus, and then the head was placed into gentamicin solution with an exposure time of 60 minutes (Figure 8).

\section{Results and discussion}

Comparison of four types of treatment of the femoral bone heads is carried out. It was revealed that the perforated femoral bone head, treated on the Lobator apparatus and soaked in gentamicin for 60 minutes, had the greatest influence on S. aureus activity.

The perforated, treated, gentamicin-soaked femoral bone head was compared with the gentamicin-soaked "PerOssal" beads in terms of the effect on S. aureus strains using the agar diffusion method. 
The antibiotic release was assessed by the agar diffusion method. We used 3 bone fragments of perforated, treated, soaked in gentamicin bone head from a certain depth level (cortical, subcortical and central) with dimensions of $1 \mathrm{~cm} \mathrm{x} 0.5 \mathrm{~cm}$.

All selected allografts were placed in sterile Petri dishes $9 \mathrm{~cm}$ in diameter ( 1 bone plate per dish). The figure shows the process of femoral bone head crushing with use of oscillator saw (Figure 9).

Figure 9 - Femoral bone head crushing with use of oscillator saw

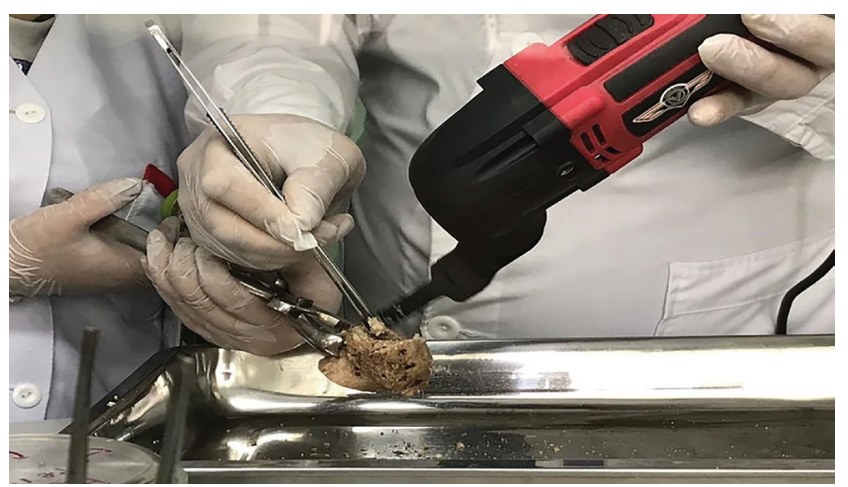

The study results indicate the presence of antimicrobial activity in all groups [13]. However, allografts perforated according to the developed technique showed a higher zone of bacteria growth inhibition in comparison with whole femoral bone heads. This fact testifies to the effectiveness of the developed device. The device contributes to the efficient formation of evenly distributed channels in the bone allograft array. The channels are intended for uniform impregnation of bone allograft spongiosis with solutions, as well as for increasing the allograft surface area for bone tissue new grow [14]. Uniform channeling facilitates deep soaking of bone graft spongy tissue with solutions of medicinal substances [15].

\section{Conclusion}

A device for bone allograft channeling with the subsequent impregnation of medicinal substances into the bone tissue is effective. The device is a rectangular metal structure, on two side walls of which there are threaded holes for brackets. They are necessary for firm fixation of the bone allograft inside the base. The other two walls have through channels located at the same distance from each other over the entire surface of the wall. The channels are $10 \mathrm{~mm}$ thick to prevent drill deflection. In the bone allograft, multiple through channels oriented from two perpendicular sides are formed, intended for subsequent uniform bone tissue impregnation.

1) The device has through channels that fix the drill direction, which eliminates the "cleaver effect" with the danger of bone wedging and cracking.

2) Wall thickness with $10 \mathrm{~mm}$ guide channels avoids the smallest deviations of the drill during channel formation.

3) The bone graft is securely fixed in the device, which avoids displacement of the bone during the bone canal formation.

4) The use of the device makes it possible to neglect the bone unevenness due to the graft fixation.

5) The presence of the guide channels of the device ensures the evenness, parallelism of the channels at the same distance, as well as the absence of intersection of the channels drawn from both sides. This has a positive effect on the strength of the allograft and on the impossibility of cavity forming, in the place of which a void defect can form during the new grows of bone tissue.

Thus, the proposed device makes it possible to improve the quality of the bone graft channeling by forming multiple non-intersecting through channels precisely oriented from two perpendicular sides at the same distance, intended for subsequent uniform impregnation of bone allograft spongy tissue with drug solutions.

Ethical aspects: The study was conducted in accordance with Directive 2010/65/EC of the European Parliament and of the Council of the European Union from September 22, 2010 on the protection of animals used for scientific purposes. All procedures are approved by the Ethical committee of the Karaganda State Medical University (Protocol No. 13 from $25 / 09 / 2017)$. Anesthesia was done by inhalation and intravenous. The object of the study were 150 rabbits, 6-8 months old with weight 3000.0-3500.0 grams. The used rabbits were from the vivarium of Karaganda medical university.

Disclosures: There is no confict of interest for all authors.

Acknowledgements: None.

Funding: The research was funded by the Science Committee of the Ministry of Education and Science of the Republic of Kazakhstan (Grant No. ??05133674).

\section{References}

1. Bozhkova SA, Novokshonova AA, Konev VA. Current trends in local antibacterial therapy of periprosthetic infection and osteomyelitis [In Russian]. Traumatology and Orthopedics of Russia. 2015;(3):92-107. https://doi.org/10.21823/2311-2905-2015-0-3-92-107

2. Witse E, Persen L, Benum P, Bergh K. Cortical allograft as a vehicle for antibiotic delivery. Acta Orthop. 2005;76(4):481-86. https:// doi.org/10.1080/17453670510041457

3. Witse E, Persen L, Benum P, Bergh K. Release of netilmicin and vancomycin from cancellous bone. Acta Orthop Scand. 2002;73(2): 199-205. https://doi.org/10.1080/000164702753671812

4. Khoo PP, Michalak KA, Yates PJ, Megson SM, Day RE, Wood DJ. Iontophoresis of antibiotics into segmental allografts. $J$ Bone Joint Surg Br. 2006;88(9):1149-57. https://doi.org/10.1302/0301-620X.88B9.17500

5. Wits0 E, Persen L, Benum P, Bergh K. Release of netilmicin and vancomycin from cancellous bone. Acta Orthop Scand. 2002;73(2):199205. https://doi.org/10.1080/000164702753671812

6. Mouzopoulos G, Kanakaris NK, Kontakis G, Obakponovwe O, Townsend R, Giannoudis PV. Management of bone infections in adults: the surgeon's and microbiologist's perspectives. Injury. 2011;42(5):18-23. https://doi.org/10.1016/S0020-1383(11)70128-0

7. Hanberger H, Edlund C, Furebring M, Giske C, Melhus A, Nilsson LE, et al. Rational use of aminoglycosides - review and recommendations by the Swedish Reference Group for Antibiotics (SRGA). Scand J Infect Dis. 2013;45(3):161-75. https://doi.org/10 .3109/00365548.2012.747694 
8. Vinogradova TP, Lavrischeva GI. Regeneration and bone transplantation [In Russian]. Moskow: Medicine; 1974.274 p.

9. Lavrischeva GI, Onoprienko GA. Morphological and clinical aspects of reparative regeneration of supporting organs and tissues [In Russian]. Moscow: Medicine. 1996. 195 p.

10. Ostroverkhov GE, Bomash YuM, Lubotsky DN. Operative surgery and topographic anatomy [In Russian]. Kursk; Moscow: Litera; 1996: p.720.

11. Kluin OS, van der Mei HC, Busscher HJ, Neut D. Biodegradable vs non-biodegradable antibiotic delivery devices in the treatment of osteomyelitis. Expert Opin Drug Deliv. 2013;10(3):341-51. https://doi.org/10.1517/17425247.2013.751371

12. Tuleubaev BE, Saginova DA, Saginov AM, Koshanova AA, Tashmetov ER. Minimally invasive method for the treatment of chronic post-traumatic osteomyelitis long tubular bones with damage to the bone marrow canal and a device for it implementation. Patent for invention No. 34571 dated 09/11/2020.

13. Tuleubaev BE, Saginova DA, Koshanova AA, Tashmetov ER, Saginov AM, Belyaev AM. Antibiotic impregnation of bone allograft: microbiological comparative analysis. Surgery News. 2019.5:489 - 495. https://doi.org/10.18484/2305-0047.2019.5.489

14. Tuleubaev BE, Kamyshansky EK, Azimova SD, Tashmetov ER, Koshanova AA, A histolic and hestomorphometric analysis of bone tissue regeneration with perforated bone allograft in rabbit femur defect. Open Access Macedonian Journal of Medical Science. 202;9(A):12-18. https://doi.org/10.3889/oamjms.2021.5588

15. Winkler H, Janata $\mathrm{O}$, Berger C, Wein W, Georgo-poulos A. In vitro release of vancomycin and tobramycin from impregnated human and bovine bone grafts. J Antimicrob Chemother. 2000;46(3):423-28. https://doi.org/10.1093/jac/46.3.423 